\title{
Simulation of incoherent ion effects in electron storage rings
}

\author{
J. Calvey $\odot^{*}$ and M. Borland $\odot$ \\ Advanced Photon Source, Argonne National Laboratory, 9700 South Cass Avenue, \\ Argonne, Illinois 60439, USA
}

(Received 20 September 2021; accepted 9 November 2021; published 3 December 2021)

\begin{abstract}
Ion trapping occurs when a negatively charged beam ionizes residual gas inside an accelerator vacuum chamber, and the resulting ions become trapped in the beam potential. In addition to the well-understood coherent instability, trapped ions can cause incoherent effects, such as emittance growth and tune spread. Typically, simulation of ion effects is done using a weak-strong model, in which the ions are modeled using macroparticles, but the beam is assumed to be a fixed Gaussian distribution, with only centroid motion allowed. This type of model necessarily neglects intra-bunch effects. Recently, a residual gas ion modeling capability has been incorporated into the particle tracking code ELEGANT. Both the beam and ions are modeled using macroparticles, so incoherent effects can be studied. The code models ion generation, movement of ions between bunches, and beam-ion interactions. It has also been parallelized and can be used in combination with other ELEGANT elements. The code has been used to study ion instability in the present APS storage ring. Once several important effects are included (multiple ionization, transverse impedance, and charge variation between bunches), the simulations show good agreement with the measured data.
\end{abstract}

DOI: 10.1103/PhysRevAccelBeams.24.124401

\section{INTRODUCTION}

Electron storage rings for synchrotron radiation sources generally aim to deliver high-current and low-emittance electron beams, to maximize the brightness of the photon beams produced. In order to maintain low emittance at high current, potential collective instabilities must be anticipated and mitigated.

One particular source of concern is ion-driven instability. Residual gas molecules can be ionized by the beam and the resulting ions can couple with the beam motion. This leads to a quickly growing coherent transverse instability, usually in the vertical plane. The strength of the instability is proportional to the average beam current and inversely proportional to the beam size [1]. In addition, trapped ions can cause incoherent effects, such as emittance growth and tune spread. These are generally less well understood than the coherent instability.

Ion instability was initially observed in its "conventional" form, in which the ion density and instability amplitude build up over many turns [2-4]. Using gaps between bunch trains, to give the ions time to clear out, is generally effective against the conventional ion instability [5]. Even with

\footnotetext{
*jcalvey@anl.gov
}

Published by the American Physical Society under the terms of the Creative Commons Attribution 4.0 International license. Further distribution of this work must maintain attribution to the author(s) and the published article's title, journal citation, and DOI. clearing gaps, one can still have a fast ion instability [6-10], which builds up over a single bunch train. Typically, this instability can be suppressed by transverse feedback.

With the use of train gaps and feedback, ion effects are generally well controlled in present-day synchrotron radiation sources. However, the potential for ion instability needs to be reevaluated for next generation light sources. These machines will have high-current and ultralow emittance, and thus a high instability growth rate is expected. In addition, because of their extremely demanding emittance requirements, they will be especially sensitive to beam size blowup caused by incoherent ion effects. This is of particular concern because significant emittance growth will change the ion-trapping criteria [2], potentially trapping more ions and leading to further emittance growth and/or instability.

The APS-upgrade is a fourth generation light source currently under development at Argonne National Laboratory [11], with a design emittance of $42 \mathrm{pm}$ at $6 \mathrm{GeV}$. Simulations predict a strong coherent instability for 324 bunch mode, which we plan to mitigate with a compensated gap scheme [12]. However, even if the coherent instability is damped, incoherent effects such as emittance growth may still be an issue.

In most ion simulation codes (e.g., [13-16]), the ions are modeled using many macroparticles, but the beam is rigid, with only centroid motion allowed, and an assumed Gaussian field. These are sometimes referred to as "weakstrong" codes. Modeling incoherent effects requires a 
"strong-strong" code, i.e., to model both beam and ions with macroparticles $[6,12,17,18]$. These simulations tend to be very computationally intensive.

To address this issue, the ioneffects element has been added to the particle tracking code ELEGANT $[19,20]$. This approach has a few advantages: ELEGANT is massively parallelized [21] and the beam is already modeled with macroparticles. It also allows for self-consistent simulation of ion effects in combination with other ELEGANT capabilities, such as beam impedance and feedback.

This paper is divided into two parts. The first (Sec. II) describes the ioneffects element and its features. The second (Sec. III) shows simulations for the present APS storage ring, and compares them to measurements. This benchmarking effort helps give us confidence in the code, and in our predictions for the APS-U, which will be reported in a future publication.

\section{DESCRIPTION OF THE IONEFFECTS ELEMENT}

The ioneffects element was designed to be as flexible as possible, allowing the user to specify the gas species, pressure profiles, interaction points (IPs), and bunch pattern. Performing ion simulations involves the following steps: (i) Prepare a file describing the ion properties, i.e., the mass, ionization cross section, and source of each ion. The source can be either a gas molecule (for single ionization) or another ion (for multiple ionization, see Sec. IID). (ii) Prepare a file giving the gas pressure around the ring, for the source gases described in the ion properties file. Each gas has an independent pressure profile, which allows for modeling variation in outgassing rates and pumping effectiveness. (iii) Insert ioneffects elements in the lattice. This can be performed using the insert_elements command or manually by editing the lattice file. Each ioneffects element represents the ions present in a segment of the accelerator, as described in Sec. II A. (iv) Insert an ion_effects command after the run_setup command in the ELEGANT command input file. Numerical parameters (such as the number of macroions generated, boundaries of the transverse region where ions are tracked, etc.) are specified here. (v) Generate a bunched beam, using either the bunched_beam command or providing an externally generated beam to the sdds_beam command. Note that this allows for an arbitrary bunch pattern.

For each bunch passage, an ioneffects element first advances existing ions to reflect ballistic motion during the gap between bunches and eliminates ions that are outside of user-specified boundaries. It then generates new ions (Sec. II A), using the local pressure of each gas species and the provided cross sections. Finally, it applies kicks from the electron bunch to the ions (Sec. II B) and from the ions to the electrons (Sec. II C).

\section{A. Ion generation}

The line density of ions generated by a single bunch in a single pass is

$$
\lambda_{\text {ion }}=\sigma_{\text {ion }} \frac{P}{k_{B} T} N_{b},
$$

where $\sigma_{\text {ion }}$ is the ionization cross section, $P$ is the pressure, $k_{B}$ is the Boltzmann constant, $T$ is the temperature, and $N_{b}$ is the bunch population. Ions are modeled as macroparticles that represent, statistically, a large number of ions. In particular, each "macro-ion" represents the motion of ions with total charge

$$
Q_{\mathrm{mac}}[C]=\frac{10^{-22} e}{7.5 \times 10^{-3} k_{B}} \frac{\sigma_{\mathrm{ion}} P N_{b} L_{\mathrm{eff}}}{n_{\mathrm{mac}} T} .
$$

Here $\sigma_{\text {ion }}$ has units of $\mathrm{Mb}, P$ has units of Torr, $k_{B}=1.38 \times 10^{-23} \mathrm{~J} / \mathrm{K}, e$ is the electron charge, $L_{\mathrm{eff}}$ is the effective length of the ion element (in meters), and $n_{\text {mac }}$ is the number of macroparticles generated (specified by the user). The numerical factors $\left(10^{-22}\right.$ and $\left.7.5 \times 10^{-3}\right)$ are simply for conversion of units. The effective length $L_{\text {eff }}$ is the segment of the ring represented by the ioneffects element, which starts half way to the previous element, and ends half way to the next one. By increasing the parameter $n_{\text {mac }}$, the user can obtain a better statistical representation of the ions at the expense of greater running time.

The initial ion transverse distribution follows the bunch distribution, which is assumed to be Gaussian.

\section{B. Beam-ion kicks}

The kick on the ions from the beam is calculated using the Basetti-Erskine formula [22], which assumes the beam is Gaussian in both transverse dimensions. The change in momentum of an ion due to the bunch passage is

$$
\begin{aligned}
\Delta p_{y}+i \Delta p_{x}= & \frac{c N_{b} r_{e} m_{e}}{\gamma} \sqrt{\frac{2 \pi}{\sigma_{x}^{2}-\sigma_{y}^{2}}}\left[\mathrm{w}\left(\frac{x+i y}{\sqrt{2\left(\sigma_{x}^{2}-\sigma_{y}^{2}\right)}}\right)\right. \\
& \left.-\exp \left(\frac{-x^{2}}{2 \sigma_{x}^{2}}-\frac{y^{2}}{2 \sigma_{y}^{2}}\right) \mathrm{w}\left(\frac{\frac{\sigma_{y}}{\sigma_{x}} x+i \frac{\sigma_{x}}{\sigma_{y}} y}{\sqrt{2\left(\sigma_{x}^{2}-\sigma_{y}^{2}\right)}}\right)\right],
\end{aligned}
$$

where $c$ is the speed of light, $N_{b}$ is the bunch population, $r_{e}$ is the classical electron radius $\left(2.82 \times 10^{-15} \mathrm{~m}\right), m_{e}$ is the electron mass, $\gamma$ is the relativistic factor ( $\sim 1$ for the ions), $\sigma_{x, y}$ are the horizontal and vertical beam sizes, $\mathrm{w}$ is the complex error function, and $x$ and $y$ are the distance from the ion to the bunch center. 
One needs to be careful when evaluating this expression numerically. If $\sigma_{x}<\sigma_{y}$, the calculation must be done in a rotated frame, with $x$ and $y$ swapped. Also, if the imaginary part of the argument of $\mathrm{w}$ is large and negative (in particular, if $\sigma_{x} \approx \sigma_{y}, y<0$ ), the two terms in the brackets of Eq. (3) become very large, though their difference remains small. Eventually, one can run into the limit of machine precision. When this happens the calculation is no longer valid, resulting in unphysically large beam kicks. Fortunately, since the kick is known physically to be symmetric for $+y$ and $-y$, this problem can be avoided simply by doing all calculations with $y>0$ and multiplying the result by -1 when appropriate.

\section{Ion-beam kicks}

For the simulations shown in this paper, Eq. (3) is also used to calculate the kick that the ion cloud gives to electrons in the bunch. In this case, $\sigma_{x}$ and $\sigma_{y}$ are now the standard deviations of the ion positions. It should be noted that the ion distribution is not in general Gaussian, though calculations by L. Wang et al. [14] have shown that the field from a typical ion distribution does resemble that of a Gaussian.

One potential pitfall of this method is that ions that are far from the beam center (e.g., on their way to being lost on the chamber wall) will artificially inflate the calculation of the ion sigmas. To mitigate this problem, only ions that are within five beam sigma of the beam centroid (in both planes) are counted in the calculation.

Additional ion-beam kick methods, where the ion distribution is fit using multiple Gaussian or Lorentzian functions, have been added to the code; they will be described in a future paper.

\section{Multiple ionization}

Ions that are trapped in the beam's potential will continue to interact with the beam and can become further ionized. Multiply ionized molecules tend to be unstable and will often dissociate into other molecules and/or constituent atoms. Either way, the charge or mass ratio of the resulting particle(s) will be changed and it or they may no longer be trapped. Also, the resulting ions can be given additional kinetic energy. On the other hand, if the ion remains trapped, it will have a bigger effect on the beam due to its increased charge. Multiple ionization has been observed at the CERN electron-positron accumulator [23].

Multiple ionization is inherently a more complicated process than single ionization. For example, in a paper studying $\mathrm{CO}_{2}^{+}$, the authors list 13 possible multiple ionization processes [24]. Fortunately, we are only interested in the resulting ions. In the case of $\mathrm{CO}_{2}^{+}$, the only products with significant cross sections are $\mathrm{CO}_{2}^{2+}, \mathrm{C}^{+}$, and $\mathrm{O}^{+}$.
We are not aware of any database or review paper that lists multiple ionization cross sections for many ions, so the numbers for each ion need to be tracked down separately. In addition, these measurements are typically only done up to the $\mathrm{keV}$ scale. Fortunately, these can be extrapolated to high energy using a formula originally derived by Hans Bethe $[25,26]$ :

$$
\sigma_{B}=\frac{8 \pi a_{0}^{2}}{m_{e} v^{2} / R}\left(M_{0}^{2}\left[\ln \left(\frac{\beta^{2}}{1-\beta^{2}}\right)-\beta^{2}\right]+C_{0}^{2}\right) .
$$

Here $a_{0}$ is the Bohr radius $\left(0.52 \times 10^{-10} \mathrm{~m}\right), m_{e}$ is the electron mass, $v$ is the velocity of the electron, $R$ is the Rydberg energy (13.61 eV), $\beta=v / c$, and $M_{0}^{2}$ and $C_{0}$ are constants that depend on the molecule. Equation (4) is valid as long as the incident electron energy is much higher than the binding energy of electrons in the molecule. Thus the constants $M_{0}^{2}$ and $C_{0}$ can be inferred from the low-energy measurements and used to extrapolate to high energy.

Table I lists the cross sections for several multiple ionization processes at $7 \mathrm{GeV}$ and gives a reference for each. Only processes with a significant cross section are listed.

The ioneffects element supports multiple ionization. In the ion properties file, one can define the source for a given ion (e.g., $\mathrm{C}^{+}$) to be another ion (e.g., $\mathrm{CO}^{+}$). In this case, each source macro-ion has a chance of being multiply ionized by a passing bunch. The probability of multiple ionization is the ionization cross section for this process multiplied by the $2 \mathrm{D}$ beam density at the macro-ion position. Specifically, it is

$$
P_{m i}=\frac{\sigma_{m i} N_{b}}{2 \pi \sigma_{x} \sigma_{y}} \exp \left[\frac{-\left(x_{b}-x_{i}\right)^{2}}{2 \sigma_{x}^{2}}-\frac{\left(y_{b}-y_{i}\right)^{2}}{2 \sigma_{y}^{2}}\right],
$$

where $\sigma_{m i}$ is the multiple ionization cross section, $N_{b}$ is the bunch population, $\sigma_{x}$ and $\sigma_{y}$ are the horizontal and vertical beam sizes, $x_{b}$ and $y_{b}$ are the beam centroids, and $x_{i}$ and $y_{i}$ are the ion coordinates. If multiple ionization occurs, the macro-ion is simply converted to the new species. Presently, this method does not allow for dissociation into

TABLE I. Cross sections for several multiple ionization processes.

\begin{tabular}{lcc}
\hline \hline Process & $\sigma(7 \mathrm{GeV}) \mathrm{Mb}$ & Reference \\
\hline $\mathrm{H}_{2}^{+} \rightarrow \mathrm{H}^{+}$ & 0.48 & {$[27]$} \\
$\mathrm{CO}^{+} \rightarrow \mathrm{C}^{+}$ & 1.22 & {$[28]$} \\
$\mathrm{CO}^{+} \rightarrow \mathrm{O}^{+}$ & 1.34 & {$[28]$} \\
$\mathrm{CO}_{2}^{+} \rightarrow \mathrm{C}^{+}$ & 0.23 & {$[24]$} \\
$\mathrm{CO}_{2}^{+} \rightarrow \mathrm{O}^{+}$ & 0.22 & {$[24]$} \\
$\mathrm{CO}_{2}^{+} \rightarrow \mathrm{CO}_{2}^{2+}$ & 0.64 & {$[24]$} \\
$\mathrm{N}_{2}^{+} \rightarrow \mathrm{N}^{+}$ & 3.6 & {$[29]$} \\
\hline \hline
\end{tabular}




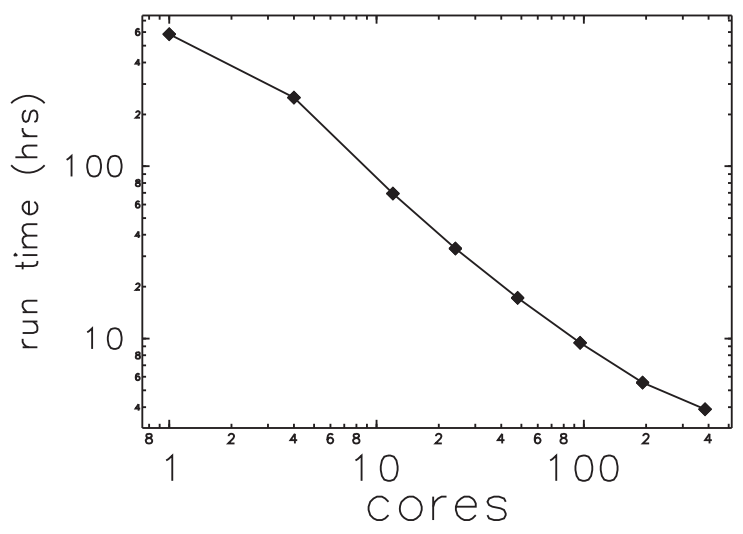

FIG. 1. Run time vs number of processors for a typical APS simulation (1000 turns).

multiple ions at once, but these processes generally have much lower cross sections.

\section{E. Parallelization}

The ioneffects element has been parallelized using the message passing interface (MPI) [30] library, and so is fully compatible with PELEGANT[21]. Parallel tracking of multibunch beams is an existing feature in PELEGANT, which we made use of here. To allow for the greatest use of parallel resources in the time-ordered modeling of bunches in the beam, each core performs the modeling of part of each bunch. Similarly, for the ions, each working processor is responsible for generation and evolution of a separate set of ions at each location. Further, the rms size of the ions is computed using MPI collective operations, so that there is no need to share the ion coordinates among processors. Statistical data from the electron bunches and ions is available in self describing data set (SDDS) files [31], which are written by the master processor using data collected from the working processors. This reduces, but does not eliminate, the interference of file operations with computations. Use of SDDS files provides a ready-made postprocessing system [32].

As shown in Fig. 1, a typical APS simulation (described below) with 1000 turns takes $\sim 600 \mathrm{~h}$ on a single core. Running PELEGANT with 12 cores reduces this time by nearly an order of magnitude; with 192 cores, another order of magnitude is gained. With 384 cores, the run time for this simulation is only $4 \mathrm{~h}$.

\section{APS SIMULATIONS}

Ion instability simulations have been run for the present APS storage ring. There are several motivations for this work. First, it allows us to study the impact of including different effects in the simulation, such as multiple ionization, transverse impedance, and charge variation. It is also interesting to understand why ion instability is not observed in present APS, though it is predicted to occur by both theory and weak-strong simulations. Given that APS is an existing ring available for both new experiments and data mining, these studies allow testing and validating the code, giving us greater confidence in our predictions for the APS-U.

\section{A. Baseline simulation}

APS operates for x-ray users with $100 \mathrm{~mA}$ stored current. The dominant operating mode features 24 equally spaced bunches. In this mode, the high-bunch charge and large bunch spacing prevent ions from being trapped in the first place. We are thus more interested in modeling standard 324 bunch operation at the APS. Basic parameters for this mode are given in Table II.

For these simulations, each bunch is modeled with 10000 macroparticles. One macro-ion of each species is generated every bunch passage. These numerical parameters were checked for convergence.

To keep run times reasonable, the lattice file uses matrix elements (SBEN, QUAD, and SEXT), with third-order concatenation. Since we are simulating the onset of instability rather than nonlinear dynamics, this is deemed a reasonable compromise. The simulations were run for 2000 turns. This was empirically found to be enough time for the instability to reach equilibrium, in most cases.

When choosing the location of ioneffects elements, it is important to ensure that the beta functions at the elements are representative of the lattice. For our case, we used 11 elements, spaced roughly evenly around the ring. The APS ring lattice consists of 40 nearly identical sectors. Since 40 and 11 have no common divisor, each ioneffects element will sample a different part of the lattice. Increasing the number of elements did not significantly change the results, as long as they were evenly spaced and had no common divisor with 40 .

The gas pressure is assumed to be a flat $0.5 \mathrm{nTorr}$ around the ring. The gas composition is $65 \% \mathrm{H}_{2}, 11 \% \mathrm{H}_{2} \mathrm{O}, 16 \%$ $\mathrm{CO}$, and $8 \% \mathrm{CO}_{2}$. The Gaussian method is used for calculating both the beam-ion and ion-beam kicks, as described above.

TABLE II. Nominal beam parameters for 324 bunch mode.

\begin{tabular}{lc}
\hline \hline Parameter & Value \\
\hline Number of bunches & 324 \\
Beam energy & $7 \mathrm{GeV}$ \\
Revolution time & $3.68 \mu \mathrm{s}$ \\
Beam current & $100 \mathrm{~mA}$ \\
Bunch charge & $1.14 \mathrm{nC}$ \\
Bunch spacing & $11.4 \mathrm{~ns}$ \\
Horizontal emittance & $2.5 \mathrm{~nm}$ \\
Vertical emittance & $0.033 \mathrm{~nm}$ \\
Horizontal chromaticity & 3 \\
Vertical chromaticity & 3 \\
\hline \hline
\end{tabular}




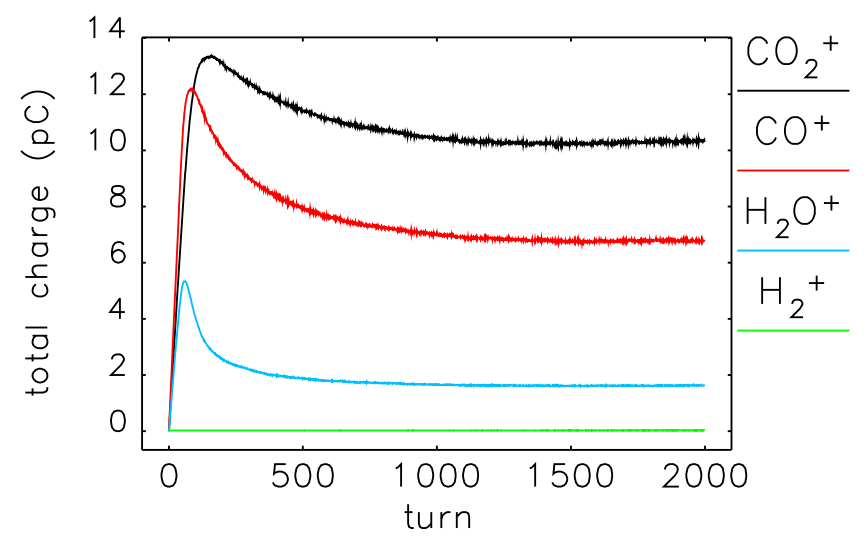

FIG. 2. Total charge of each ion species in the baseline APS simulation.

The results of this simulation are shown in Figs. 2 through 5. In general, one sees the standard behavior of ion instability - a fast initial growth that starts to slow once the instability amplitude reaches a significant fraction of the beam size. This corresponds to a reduction in the ion density, as ions are shaken out by the beam motion. This beam shaking limits the final instability amplitude to around one beam sigma.

Specifically, Fig. 2 shows the total charge (summed around the ring) for each ion species. Since $\mathrm{H}_{2}^{+}$is not trapped anywhere in the ring, its density is essentially zero. $\mathrm{H}_{2} \mathrm{O}^{+}$is only trapped in certain locations, and is mostly shaken out when the beam becomes unstable, as lighter ions are more susceptible to beam shaking. Initially, $\mathrm{CO}^{+}$is the dominant species, due to its high partial pressure, but its density is also limited by shaking. In the end, the heaviest ion, $\mathrm{CO}_{2}^{+}$, dominates.

Figure 3 shows the simulated ion density vs turn, for two different IPs. They exhibit different behavior because the critical mass [2] is different: $A_{\text {crit }}=2.6$ at IP2 and 6.6 at IP6. Effectively, this means that ions are more easily shaken out at IP6.

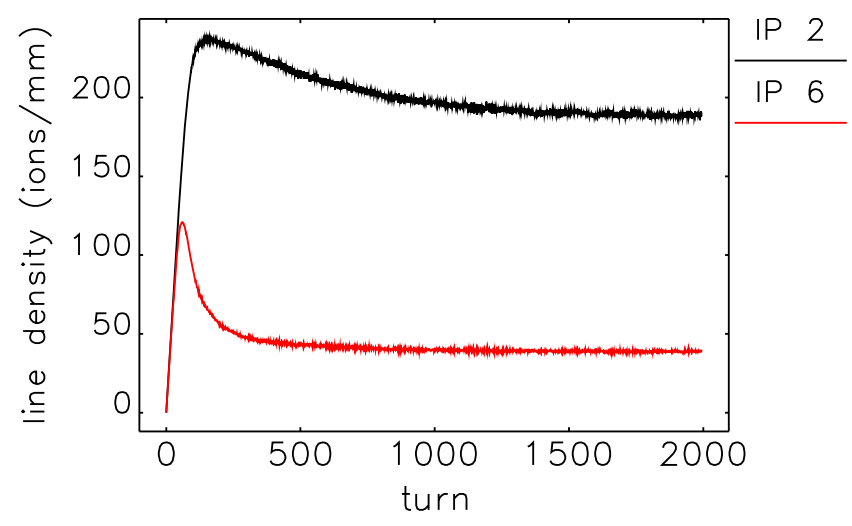

FIG. 3. Ion density at different interaction points in the baseline APS simulation.

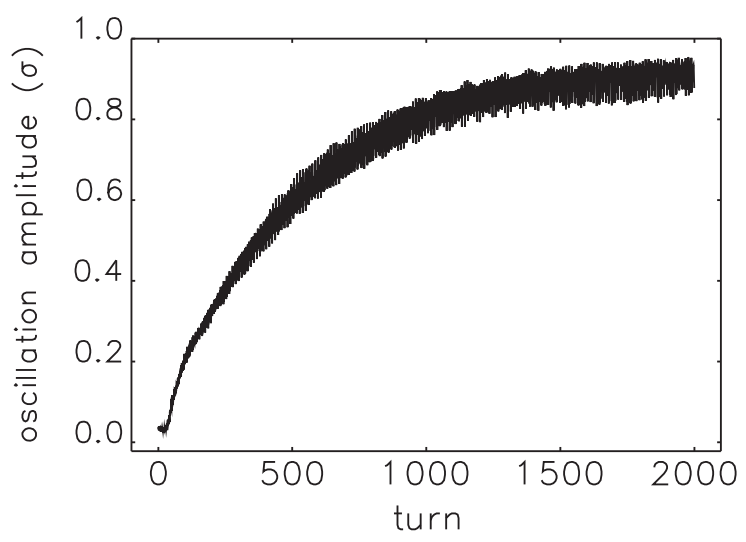

FIG. 4. Vertical instability amplitude for the baseline simulation.

Figure 4 plots the vertical instability amplitude, as a fraction of the vertical beam size. There is a fast initial growth that slows once the instability amplitude reaches $\sim 20 \%$ of the beam size. The amplitude after 2000 turns is $\sim 0.85 \sigma_{y}$. The qualitative behavior of the instability is consistent with results from a weak-strong code [12].

A key signature of ion instability is peaks in the lower betatron sidebands at a characteristic ion frequency [14], given by Eq. (6). Here $N_{e}$ is the bunch population, $r_{p} \approx$ $1.5 \times 10^{-18} \mathrm{~m}$ is the classical proton radius, $S_{b}$ is the bunch spacing, $\sigma_{x}$ and $\sigma_{y}$ are the horizontal and vertical beam sizes, $A$ is the ion mass number, and $Q$ is the charge number of the ion (=1 for a singly ionized molecule).

$$
\omega_{i, y} \approx c\left(\frac{4 N_{e} r_{p} Q}{3 A S_{b}\left(\sigma_{x}+\sigma_{y}\right) \sigma_{y}}\right)^{1 / 2}
$$

Figure 5 shows the amplitude of these sidebands in the baseline simulation. These are calculated by doing a fast fourier transform (FFT) of the vertical position of each bunch, and picking out the peaks at the lower betatron sidebands: $f_{0}\left(n-\nu_{y}\right)$, where $n$ is an integer, $f_{0}$ is the

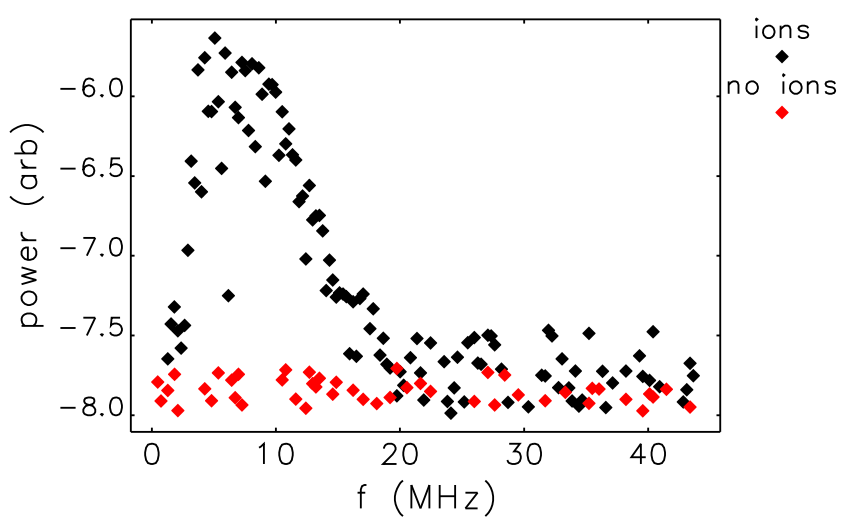

FIG. 5. Amplitude of lower vertical betatron sidebands in the baseline simulation, compared to a simulation with no ions. 


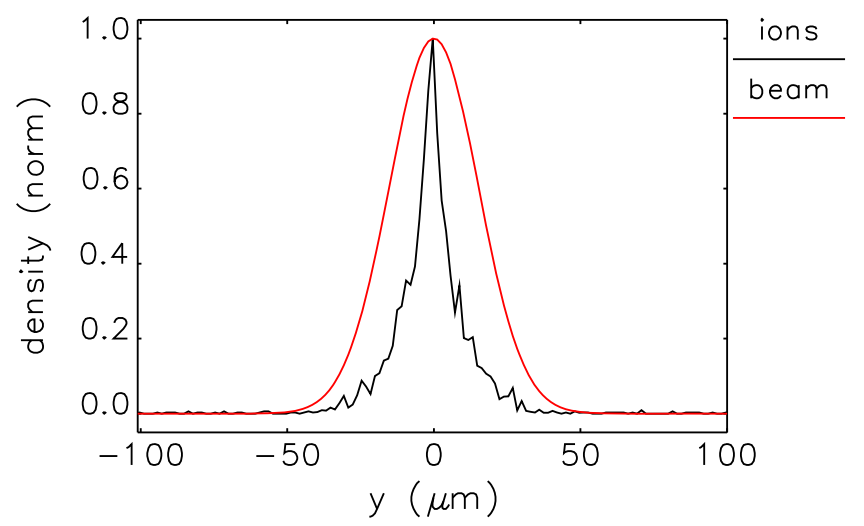

FIG. 6. Vertical ion distribution, after three turns, at IP1.

revolution frequency, and $\nu_{y}$ is the fractional vertical betatron tune. An ion peak is clearly visible from 5-10 MHz. The expected peak from Eq. (6) (averaged around the ring) is $9.0 \mathrm{MHz}$ for $\mathrm{CO}$ and $7.2 \mathrm{MHz}$ for $\mathrm{CO}_{2}$, consistent with the simulation. For the sake of comparison, the sidebands for a simulation with no ions are also shown. No elevated sidebands are observed.

One can also look at the transverse distribution of the ions. Figure 6 shows the vertical profile of the ion cloud at IP1, after three turns. The distribution is more sharply peaked than the Gaussian electron beam, which is consistent with theory [14].

\section{B. Additional effects}

This baseline simulation predicts an instability that is not actually observed in APS operation, which implies that some detail is missing from the simulation. Starting from the baseline simulation, three additional effects were incorporated into the simulation: multiple ionization (see Sec. II D), transverse impedance, and bunch-to-bunch charge variation. These effects were first simulated separately, to study their individual impact on the results, and then combined together.

\section{Transverse impedance}

Even at the low bunch charge in 324 bunch mode, headtail damping due to the short range transverse impedance can have an impact. A model for the storage ring impedance has been included in the simulation, using the ZTRANSVERSE element. This model has been well validated by storage ring measurements, such as the instability threshold vs chromaticity [33]. Note that the longitudinal impedance was not included because at such low-bunch current its effect on energy spread and bunch duration is negligible.

\section{Charge variation}

In a real machine, the charge in each bunch will not be exactly equal. Sources of charge variation include variation
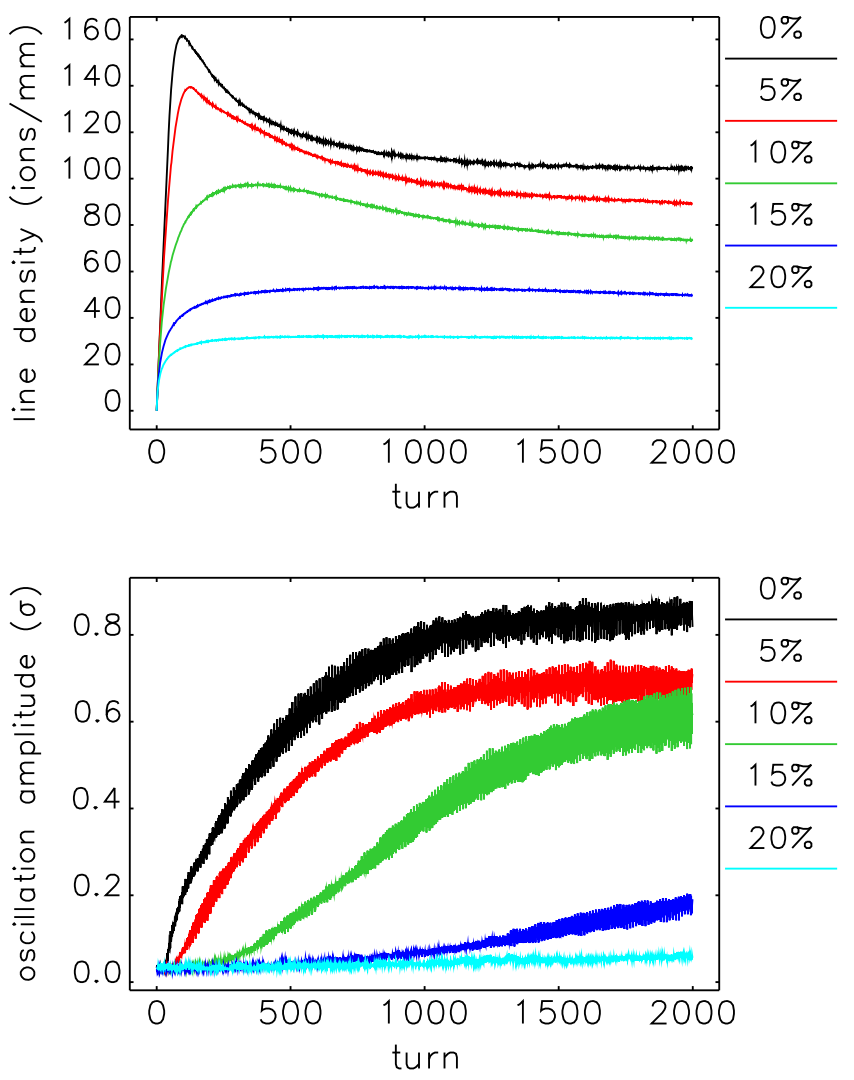

FIG. 7. Ion density (top) and instability amplitude (bottom), comparing simulations with increasing bunch charge variation.

in the charge per shot delivered by the injector, as well as time-dependent changes in the injection efficiency. Uneven bunch charge results in a variation in the focusing force seen by the ions, which can change the ion trapping criteria and may allow more of them to escape.

Since ELEGANT assumes that all beam macro-particles represent the same charge, charge variation was modeled by randomly changing the number of macro-particles in each bunch. For these simulations, a Gaussian bunch charge variation with a given sigma was used. Figure 7 compares the ion density and instability amplitude as the amount of variation is increased from $0-20 \% \mathrm{rms}$. The instability is suppressed as the charge variation is increased; by $20 \%$ rms variation it is mostly gone.

It is also notable how much the ion density is reduced by charge variation. If the variation is large enough, the assumption that the ions see an approximately constant focusing force from the beam is no longer valid, and the trapping condition [2] becomes more complicated. This suggests using an intentionally uneven fill as a possible mitigation for ion instability, which may in some circumstances be more attractive that using gaps.

\section{Results}

The effect of multiple ionization, transverse impedance, and $(10 \% \mathrm{rms})$ charge variation are summarized in Fig. 8. 

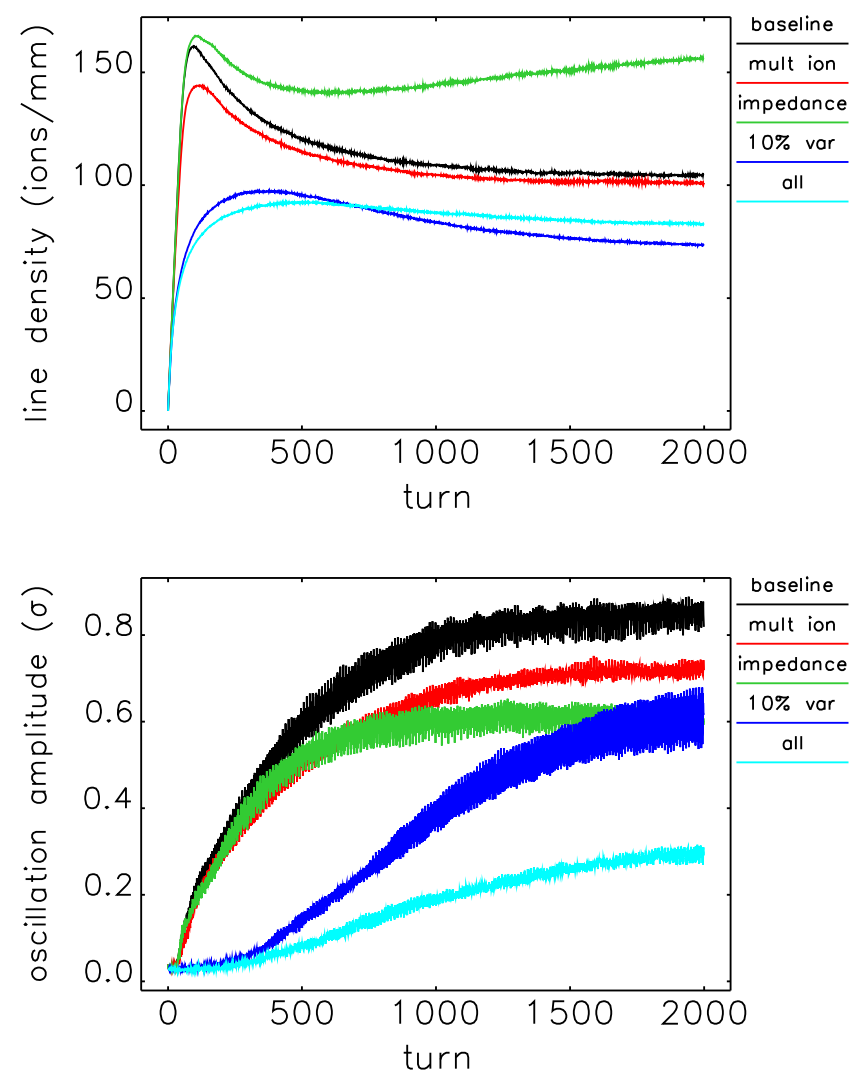

FIG. 8. Ion density (top) and instability amplitude (bottom), comparing different effects: multiple ionization (red), transverse impedance (green), and 10\% rms charge variation (blue). The result of including all three effects is shown in cyan.

When multiple ionization is added to the baseline simulation, there is a modest but noticeable reduction in the ion density and instability amplitude. The final amplitude is reduced from $\sim 0.85$ to $\sim 0.72 \sigma_{y}$. Including transverse impedance also helps limit the instability, to about $\sim 0.6 \sigma_{y}$. The ion density is not decreased in this case, since there is less electron beam motion to drive the ions into the wall.

When all three effects are included together, the instability amplitude is reduced by a factor of 3 . Of course, more charge variation would reduce this amplitude further. For the rest of this paper, all results include multiple ionization and transverse impedance.

\section{Comparison with measurements}

A long unsolved mystery at the APS is why ion instability is not seen during normal operation in 324 bunch mode (as predicted by both theory and weak-strong simulations). A significant instability would be observed primarily as a vertical emittance increase, as measured with the synchrotron light monitor. This detector averages over many turns, and hence would see a centroid instability as a beam-size increase.

To help address this question, a spectrum analyzer measurement was taken during normal operation (Fig. 9,

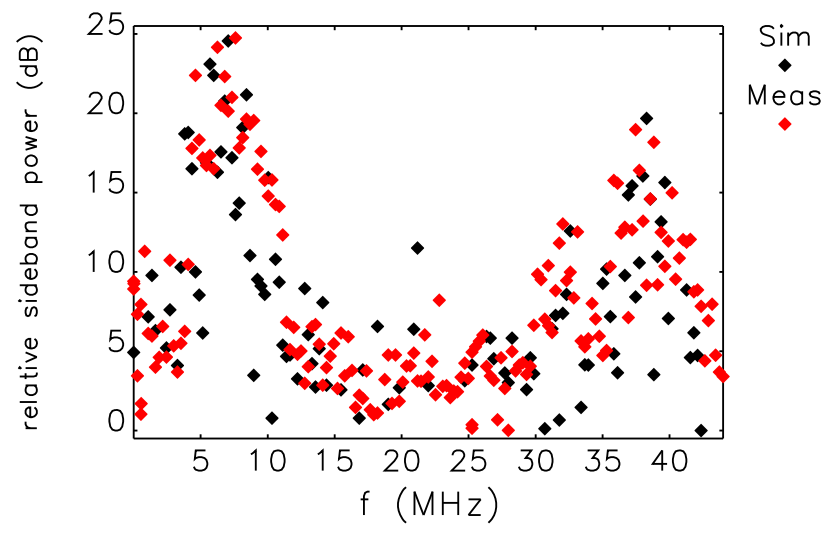

FIG. 9. Measured and simulated vertical beam spectrum for normal 324 bunch operation. The points shown are the lower vertical betatron sidebands.

red points). Here we see that there is, in fact, an observable ion peak at $\sim 7 \mathrm{MHz}$, which is approximately the average ion frequency for $\mathrm{CO}_{2}$ around the ring.

The second peak around $\sim 38 \mathrm{MHz}$ is an artifact of the uneven bunch pattern. Due to a mismatch between the charge per bunch from the injector and the required charge per bunch for 324-bunch mode, the bunch charge is usually very uneven along the train. The bunch current waveform corresponding to the measurement in Fig. 9 is shown in Fig. 10 (top). An FFT of the bunch waveform (Fig. 10, bottom) reveals a peak at $31 \mathrm{MHz}$. Effectively, the amplitude of the signal seen by the spectrum analyzer will be modulated at this frequency. This will result in sidebands at the ion frequency \pm the modulation frequency. For the case shown in Fig. 9, this results in a peak at $7 \mathrm{MHz}+31 \mathrm{MHz}=38 \mathrm{MHz}$. As explained above, this uneven fill can help mitigate the instability.

To accurately simulate this situation with ioneffects, the actual bunch pattern should be used. The script for generating a bunch train with charge variation was modified to take a measured bunch pattern as input, and generate a train with the same bunch charge distribution. This was used as input to an ELEGANT simulation, with the other input parameters the same as described above.

The results of the simulation are also shown in Fig. 9 (black points). The simulation accurately reproduces both the real ion peak at $7 \mathrm{MHz}$ and the artificial peak at $38 \mathrm{MHz}$. Because the spectrum analyzer measurement is uncalibrated, there is an arbitrary scaling in the measured waveform, which translates to an arbitrary shift in the waveform power. So while the exact values of the measured and simulated power cannot be compared, the height of the peaks relative to each other and to the noise floor can. Both show good agreement between measurement and simulation.

No significant vertical emittance increase was observed in the simulation. Taking into account the oscillation (by adding it in quadrature with the beam size), the effective 

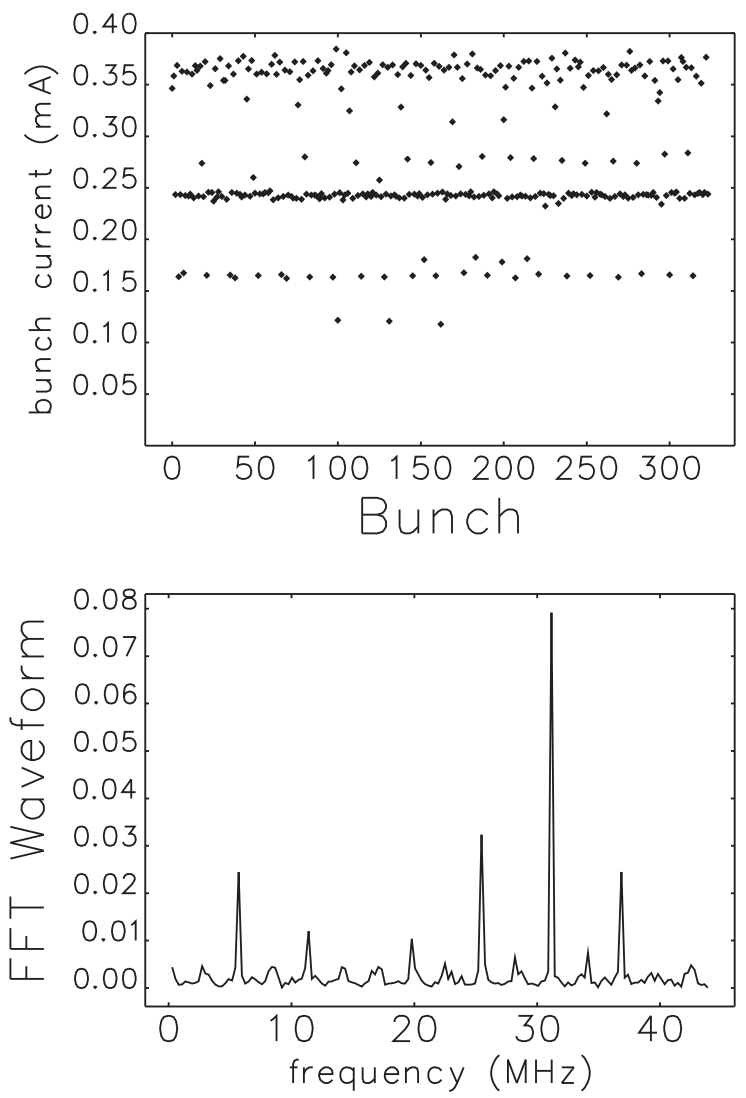

FIG. 10. Top: measured bunch pattern corresponding to Fig. 9. Bottom: FFT of this pattern.

emittance is increased by about $0.3 \%$. This is far too small to be observed by the synchrotron light monitor or have an impact on normal operations. Thus, we conclude that there actually is ion instability in the present APS, just at too small a level to observe directly.

\section{Simulations with high pressure}

While the ion instability is benign in the present APS, these results raise an interesting question: how much worse would the pressure have to be to cause a problem? To answer this, a series of simulations was run with increasing vacuum pressure around the ring: 2, 8, and 16 nTorr. All other parameters (including the gas composition) are the same as above.

The ion density for each of these cases is plotted in Fig. 11. For the most part, the density increases linearly with pressure. However, some interesting behavior can be seen in the 16 nTorr case. After a few hundred turns, the density begins to increase again. It then reaches a peak and starts to decrease.

Figure 12 plots both the vertical instability amplitude and rms beam size for each of these cases. Increasing the pressure increases the instability amplitude, up to $\sim 0.9 \sigma_{y}$ at $2 \mathrm{nTorr}, 3 \sigma_{y}$ at $8 \mathrm{nTorr}$, and $4 \sigma_{y}$ at $16 \mathrm{nTorr}$. In addition, the rms beam size of the train starts to increase at 8 nTorr

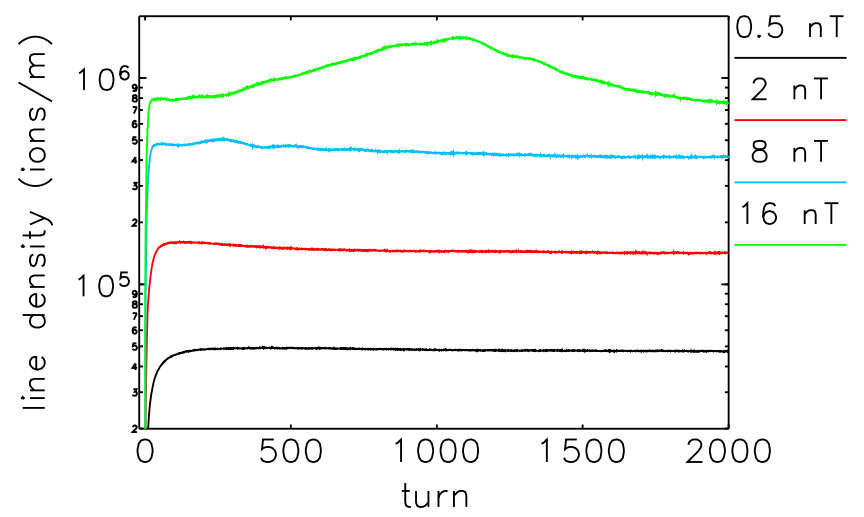

FIG. 11. Ion density for different pressure values. Note the log scale on the vertical axis.

pressure. At 16 nTorr, the beam size blowup is dramatic. Note that the oscillation amplitude is plotted in units of the initial beam size, so the scale is not affected by the beam size blowup.

Looking more closely at the 16 nTorr simulation, four different time regions can be defined, each with qualitatively different behavior. For turns $1-50$, the trapped ions cause an exponential rise in the coherent vertical instability amplitude. Between turns 50 and 300, the instability
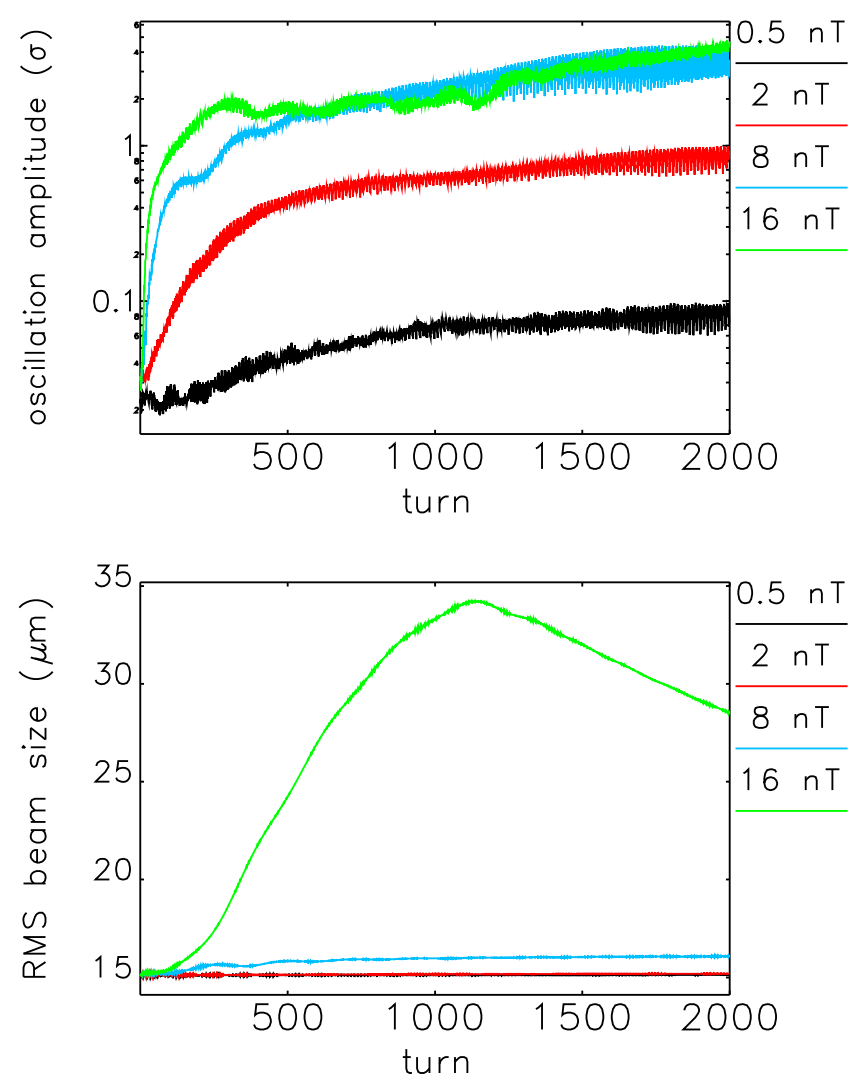

FIG. 12. Vertical instability amplitude (top) and rms beam size (bottom), for different pressure values. 

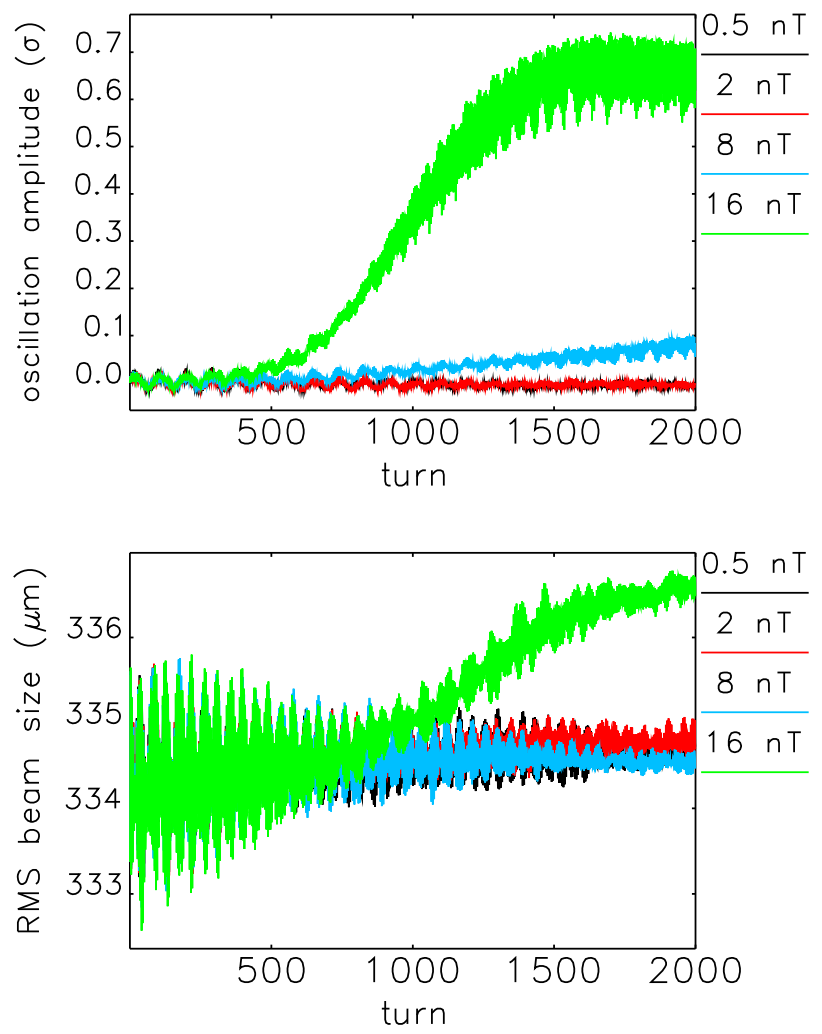

FIG. 13. Horizontal instability amplitude (top) and rms beam size (bottom), for different pressure values.

growth slows down, then saturates at $2 \sigma$, as beam motion starts to shake out the ions. From turns 300 to 1000, coherent damping transfers some of the beam motion into a large vertical beam size blowup. There is a corresponding increase in the ion density (Fig. 11), as more ions are trapped by the blown up beam size. Between turns 1000 and 2000, increased ion trapping drives the coherent instability amplitude up to $4 \sigma$. This shakes out more ions, causing a decrease in the ion density. A coherent instability also develops in the horizontal plane (Fig. 13), which contributes to the beam shaking. There is a slow decrease in the vertical beam size.

These results illustrate a dangerous scenario-the ion instability causes emittance growth, which leads to more ion trapping, further driving the instability. In the end, beam shaking of the ions still limits the instability amplitude, but at a higher value than one would expect without emittance growth. Of course, the emittance growth itself is also undesirable.

Both the oscillation and beam size blowup would be observed as effective emittance growth by the synchrotron light monitor. These two effects can be added in quadrature. The resulting emittance that would be observed as a function of pressure is shown in Fig. 14. The final effective emittance increase is about $5 \%$ for 2 nTorr, $41 \%$ for 8 nTorr, and $370 \%$ for $16 \mathrm{nTorr}$. In addition, a notable increase in the horizontal emittance is observed for the 16 nTorr case.
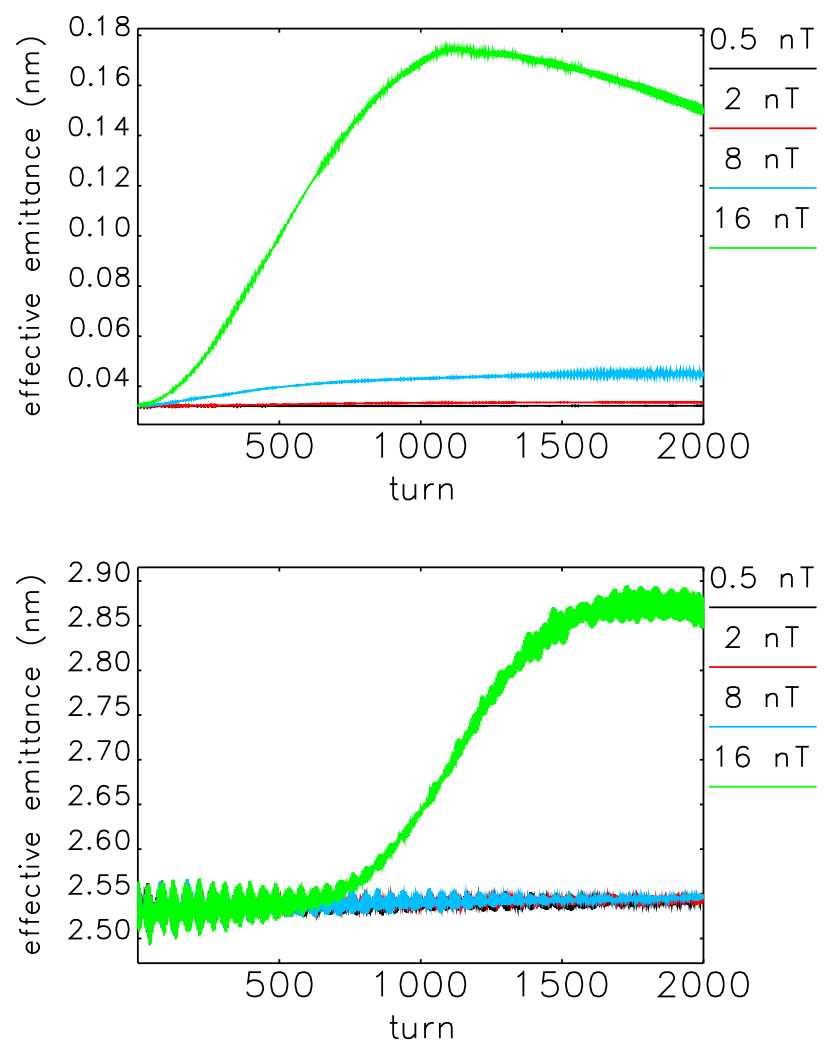

FIG. 14. Effective emittance in the vertical (top) and horizontal (bottom) planes, for different pressure values.

Consistent with operational experience, the emittance increase for $0.5 \mathrm{nTorr}$ is negligible.

\section{CONCLUSIONS}

A method of modeling the effects of residual gas ions has been developed for ELEGANT, using ion IPs implemented as a new type of beamline element, called ioneffects. The element models the generation of ions, interactions between the ions and beam, and the tracking of ions between bunch passages. It has been parallelized using MPI, which can reduce the run time by more than two orders of magnitude with enough cores. Ioneffects allows for the study of incoherent ions effects, and can be used in combination with any other ELEGANT elements (such as feedback, impedance). Application of this code to APS operational parameters has both validated the code and yielded insights into historical experience of the lack of expected ion instability.

Vertical emittance growth is observed for simulations with high pressure. This can trigger a dangerous feedback loop, as the blown up beam traps more ions, further driving the instability.

Modeling the ion instability using the ioneffects element in ELEGANT has significant advantages over weakstrong ion codes. The possibility of emittance growth can only be studied with a code that represents both the beam 
and ions with macroparticles. Similarly, ioneffects can simulate the effect of head-tail damping (and decoherence in general). We are also not aware of any other ion codes that can model an arbitrary bunch pattern. These effects were crucial in understanding the lack of measured ion instability in the present APS. We believe the ioneffects code would be similarly useful for studying coherent and incoherent ion effects in other electron storage rings.

\section{ACKNOWLEDGMENTS}

We would like to thank L. Emery, K. Harkay, and R. Lindberg for helpful discussions. This research used resources of the Advanced Photon Source, a U.S. Department of Energy (DOE) Office of Science User Facility at Argonne National Laboratory and is based on research supported by the U.S. DOE Office of ScienceBasic Energy Sciences, under Contract No. DE-AC0206CH11357. We gratefully acknowledge the computing resources provided on Bebop, a high-performance computing cluster operated by the Laboratory Computing Resource Center at Argonne National Laboratory.

[1] H. G. Hereward, Coherent instability due to electrons in a coasting proton beam, Technical Report No. CERN-71-15, 1971.

[2] Y. Baconnier and G. Brianti, The stability of ions in bunched beam machines, Technical Report No. CERN/ SPS/80-2, 1980.

[3] R. A. Pires, D. Moehl, Y. Orlov, F. Pedersen, A. Poncet, and S. van der Meer, On the theory of coherent instabilities due to coupling between a dense cooled beam and charged particles from the residual gas, in Proceedings of the 13th Particle Accelerator Conference (PAC'89) (JACoW Publishing, Chicago, 1989), pp. 800-803.

[4] S. J. Werkema, D. W. Peterson, and P. Zhou, Transverse emittance growth in the Fermilab Antiproton Accumulator with high-current antiproton stacks, in Proceedings of the 15th Particle Accelerator Conference (PAC'93), (JACoW Publishing, Washington D.C., 1993), pp. 3573-3576.

[5] D. Villevald and S. Heifets, Ion trapping in the SLAC B-factory high energy ring, Technical Report No. SLACTN-06-032, 1993.

[6] T. O. Raubenheimer and F. Zimmermann, Fast beam-ion instability. I. Linear theory and simulations, Phys. Rev. E 52, 5487 (1995).

[7] G. V. Stupakov, T. O. Raubenheimer, and F. Zimmermann, Fast beam-ion instability. II. Effect of ion decoherence, Phys. Rev. E 52, 5499 (1995).

[8] J. Byrd, A. Chao, S. Heifets, M. Minty, T. O. Raubenheimer, J. Seeman, G. Stupakov, J. Thomson, and F. Zimmermann, First Observations of a "Fast Beam-Ion Instability”, Phys. Rev. Lett. 79, 79 (1997).

[9] M. Kwon, J. Y. Huang, T.-Y. Lee, I. S. Ko, Y. H. Chin, H. Fukuma, M. Isawa, K. Ohmi, and M. Tobiyama, Experimental results on the fast beam-ion instability, Phys. Rev. E 57, 6016 (1998).
[10] J. Y. Huang, M. Kwon, T.-Y. Lee, I. S. Ko, Y. H. Chin, and H. Fukuma, Direct Observation of the Fast Beam-Ion Instability, Phys. Rev. Lett. 81, 4388 (1998).

[11] Advanced Photon Source Upgrade project preliminary design report, Technical Report No. APSU-2.01-RPT-002, 2017. https://www.aps.anl.gov/APS-Upgrade/Documents.

[12] J. Calvey and M. Borland, Modeling ion effects for the Argonne Advanced Photon Source upgrade, Phys. Rev. Accel. Beams 22, 114403 (2019).

[13] K. Ohmi, Numerical study for the two-beam instability due to ions in electron-storage rings, Phys. Rev. E 55, 7550 (1997).

[14] L. Wang, Y. Cai, T. Raubenheimer, and H. Fukuma, Suppression of beam-ion instability in electron rings with multibunch train beam fillings, Phys. Rev. ST Accel. Beams 14, 084401 (2011).

[15] L. Wang, J. Safranek, Y. Cai, J. Corbett, R. O. Hettel, T. O. Raubenheimer, J. Schmerge, and J. Sebek, Beam ion instability: Measurement, analysis, and simulation, Phys. Rev. ST Accel. Beams 16, 104402 (2013).

[16] K. Hirata, K. Ohmi, and N. Toge, Quasi-strong-strong simulations for beam-beam interactions in KEKB, in Proceedings of the 5th European Particle Accelerator Conference (EPAC'96) (JACoW Publishing, Sitges, 1996).

[17] G. Rumolo and D. Schulte, Fast ion instability in the CLIC transfer line and main linac, in Proceedings of the 11th European Particle Accelerator Conference (EPAC'08) (JACoW Publishing, 2008), pp. 655-657.

[18] C. Li, S. Tian, N. Wang, and H. Xu, Beam-ion instability and its mitigation with feedback system, Phys. Rev. Accel. Beams 23, 074401 (2020).

[19] M. Borland, elegant: A flexible SDDS-compliant code for accelerator simulation, Technical Report No. LS-287, 2000 .

[20] M. Borland et al., elegant manual, Technical Report No. LS-287, 2010.

[21] Y. Wang and M. Borland, Implementation and performance of parallelized elegant, in Proceedings of the 2007 Particle Accelerator Conference 2007 (JACoW Publishing, Albuquerque, 2007), pp. 3444-3446.

[22] M. Bassetti and G. Erskine, Closed expression for the electrical field of a two-dimensional gaussian charge, Technical Report No. CERN-ISR-TH-80-06, CERN, 1983.

[23] P. F. Tavares, Bremsstrahlung detection of ions trapped in the EPA electron beam, Part. Accel. 43, 107 (1993).

[24] E. M. Bahati, J. J. Jureta, D. S. Belic, S. Rachafi, and P. Defrance, Electron impact ionization and dissociation of CO2+ to C+ and O+, J. Phys. B 34, 1757 (2001).

[25] H. Bethe, Theory of the passage of fast corpuscular rays through matter, Ann. Phys. (N.Y.) 5, 325 (1930).

[26] M. Inokuti, Inelastic collisions of fast charged particles with atoms and molecules the Bethe theory revisited, Rev. Mod. Phys. 43, 297 (1971).

[27] J. W. Liu, An accurate total inelastic cross section for $\mathrm{H} 2+$ colliding with fast charged particles in the adiabatic approximation, Mol. Phys. 28, 343 (1974).

[28] J. Lecointre, D. S. Belic, H. Cherkani-Hassani, J. J. Jureta, and P. Defrance, A crossed-beam experiment for electron impact ionization and dissociation of molecular ions: Its application to CO+, J. Phys. B 39, 3275 (2006). 
[29] Z. Shen, E. Wang, M. Gong, X. Shan, and X. Chen, Electron-impact ionization cross sections for nitrogen molecule from 250 to $8000 \mathrm{eV}$, J. Electron Spectrosc. Relat. Phenom. 225, 42 (2018).

[30] W. Gropp, E. Lusk, N. Doss, and A. Skjellum, A highperformance, portable implementation of the MPI message passing interface standard, Parallel Comput. 22, 789 (1996).

[31] M. Borland, A self-describing file protocol for simulation integration and shared postprocessors, in Proceedings of PAC 1995, Dallas, TX, 1995 (JACoW Publishing, Dallas, 1995).
[32] R. Soliday, M. Borland, L. Emery, and H. Shang, New features in the SDDS toolkit, in Proceedings of the 2003 Particle Accelerator Conference, 2003 (JACoW Publishing, Portland, 2003), pp. 3473-3475.

[33] R. R. Lindberg and A. Blednykh, Instability thresholds for the Advanced Photon Source multibend achromat upgrade, in Proceedings of IPAC15, 2015 (JACoW Publishing, Richmond, 2015), pp. 1822-1824, https://doi.org/10.18429/JACoW-IPAC2015TUPJE077. 